\title{
AN ATM SWITCH CONTROL INTERFACE FOR QUALITY OF SERVICE AND RELIABILITY
}

\author{
Rahul Garg \\ Indian Institute of Technology, Delhi, India \\ rahul@cse.iitd.ernet.in
}

Raphael Rom

Technion, Haifa, Israel and Sun Microsystems, Palo Alto CA, USA

rom@ee.technion.ac.il

\begin{abstract}
Traditional communication switches include an embedded processor that implements both the switch control and network signalling. Such an architecture is failure-prone due to the complexity of the control software of modern network. Recently, a new approach of controlling switches through an external controller is gaining momentum, due the flexibility and reliability it affords. In addition, open control protocols and interfaces for controlling and managing networks, are now emerging as an alternative to specifications and standards. For instance, in the OpeNet project Sun Labs has designed and implemented an open, high performance ATM network control platform.

In this paper we describe the OpeNet Switch Control Interface (ONSCI)-an open local switch control protocol, for controlling an ATM switch. An ATM switch controller uses this protocol to setup or tear down virtual circuits and perform other control and management functions in an ATM switch. Important and distinguished features of this protocol are primitives for Quality of Service (QoS) management in the switch and support for fault-tolerant operation in case of failure of a switch controller. The design of these primitives is based on conceptual modeling of the switch architecture. The model is generic enough to cover a wide range of ATM switches.

The protocol was implemented and integrated with the OpeNet platform. Without going into specifics of the protocol, we describe its design principles and show how it has affected our protocol.
\end{abstract}

Keywords: ATM, GSMP, QoS, Fault Tolerance, Availability, Reliability, IP-Switching, Open Interfaces, Admission Control, GCAC, PNNI. 


\section{INTRODUCTION}

Communication switches are built from two major components: a hardware component where the data switching takes place, and a software component which provides the control mechanisms and the integration of the individual switch into a complete network. In traditional switches the control software is implemented on a dedicated processor embedded into the switch's hardware. This integration is typically tightly coupled and is tailor-made for the specific hardware and network application. Such an architecture suffers from many disadvantages such as inability to cope with the progress of processor and software technology and the inability to re-use the software.

A new trend has started recently and is gaining momentum: to devise open network architectures based on distributed systems principles. Several research groups as well as network equipment manufacturers are engaged in active pursuit of the problem. Moreover, standardization efforts have recently started by the IEEE (e.g., the P1520 working group on application programming interfaces for networks [10] and its ATM sub-working group) with a goal of defining APIs for future multimedia networks. With such an interface in place, the controller is typically detached from the rest of the hardware, need not be tightly coupled with it, the entire design allows for easy upgrading of software and lends itself to better fault tolerance.

As part of its activities, Sun Microsystems Laboratories designed and implemented OpeNet [3], an open, non-proprietary, high performance, switch independent ATM network control platform. To achieve the goal of switch independence, none of the mechanisms deployed by OpeNet rely on any particular switch. However, to be deployed for operation, the OpeNet must be interfaced with an actual ATM switch. Thus the need for a generic interface to control ATM switches was felt. This lead to the development of the OpeNet Switch Control Interface (ONSCI) [7] which is the subject of this document.

Our work has two basic distinguishing features from other works on open ATM switch control interfaces. Firstly, we provide support for managing Quality of Service (QoS) in the switch in a manner compatible with the ATM Forum specifications. Secondly, primitives for fault tolerant operation are provided to assist recovery of a switch controller after its failure. In the event of failure of a switch controller, a backup controller (or the recovering failed controller) may assume the control of the switch and after some recovery operations, starts functioning as its primary controller. Other operations supported by ONSCI are setting up and tearing down VCs, configure and manage its ports, manage its VPs and get other statistics and information related to performance monitoring. The interface uses a message exchange protocol which is client/server in nature; the switch controller sends requests to the switch which responds after processing the request. 
The Generalized Switch Management Protocol (GSMP) [14] was the first protocol developed to control ATM switches. GSMP supports only basic operation like setting up and tearing down virtual circuits, monitoring performance, and lacks the more advanced QoS functions needed in an ATM network. The second version of GSMP [15] addressed the problem of QoS in the framework of Class Based Queuing (CBQ) [6]. This model is appropriate for the Internet suite of protocols like RSVP [19], but is still inadequate (and was not intended) for ATM networks. A GSMP extension was proposed [1], in the context and framework of Xbind [11] that uses the model of schedulable region [9] to carry out admission control, but is not compatible with the specifications of the ATM Forum. The work by Evans et._al. [5] define a reasonable model of managing an ATM switch that provides QoS guarantees, but proposes only an approach and not a complete specification. None of these addresses fault tolerance issues.

The rest of the paper is organized as follows. Section 2 contains a brief overview of ONSCI design. To provide QoS support, the model of switch's resources and how the controller manages these resources is very important. This is discussed in Section 3, which is the major contribution of this paper. Section 4 details our assumptions and approach to support fault tolerance. The paper concludes in Section 5.

\section{ONSCI DESIGN}

A traditional switch of an ATM network is shown in Figure 1. It has two parts: A switching component, sometimes called the fabric or the cross-connect, a set of tables containing the forwarding information and configuration data, and a processing unit that embodies the control functions.

The ATM switch forwards its incoming cells to different output ports. This forwarding is done at very high speed using the cross-connect. The entries in the forwarding table dictate which cell should be forwarded to which port. Most of the flow control, monitoring, statistics collection is jointly done done by the hardware and software components. Other network wide operations like routing etc., are done solely by the processor. In a typical network, decisions are made by the software running in the processor and then stored in the tables to be used by the cross-connect. Such manipulation of the data structures is typically done by a tight integration between the processor, the cross-connect, and the specific manner in which the data is stored in the tables.

To overcome the deficiencies of a traditional ATM switch, a new architecture is proposed as depicted in Figure 2. An ATM node is divided into two major parts: The node controller (or switch controller) and the nodal switching subsystem (also referred to as "the switch"). The switching subsystem is essentially a very simple traditional switch where the processor need have extremely limited computational power and a small interconnect-protocol (software) module 


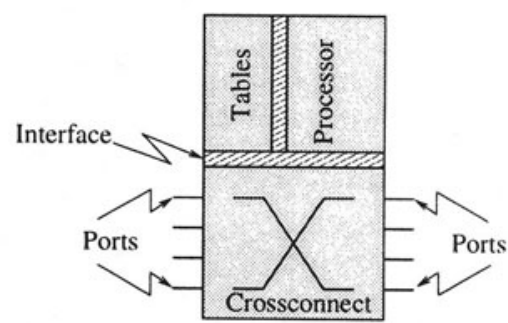

Figure 1 A traditional ATM switch

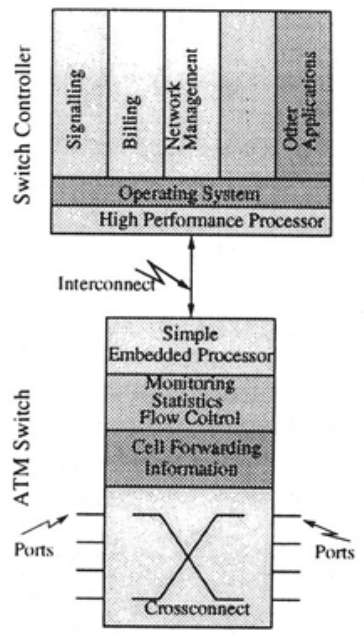

Figure 2 An ATM Node

with which it communicates with the controller. The switch is expected to have a limited capability processor, and a minimal operating system. The design of ONSCI is influenced by this assumption.

The switch controller is a generic high performance computing platform. No restrictions are imposed on its architecture. For instance, it may be a high end workstation with a generic operating system.

The switch controller sends messages to the ATM switch through a duplex interconnect. The ATM switch processes the message and sends back the response to the controller. ONSCI uses a regular ATM port for the interconnection. This makes the ATM switch uniform and enables a simpler and richer fault recovery mechanism (Section 4).

ONSCI primitives allow programming of the switch forwarding table. Thus, the controller may send and receive (signalling) messages on special VCs (VCI between 0 and 31 on VPI 0 ) of each port by diverting them to specific VCs on the ATM interconnect. The switch tables can be programmed using ONSCI to reserve resources for connections (in a manner compatible with the ATM Forum). ONSCI is not tied to one particular signalling protocol. For instance, in one configuration of the network, all the nodes may use PNNI signalling, whereas in another configuration OpeNet signalling may be used. ONSCI also supports functions that are used for operations such as monitoring, management, configuration control, link failure indications, and processing of OAM cells.

To maintain the reliability, the processor that is implemented in the switch is a very simple one, not running any complex operation but just responding 
to requests from the controller. Therefore, it is clear that the controller is the susceptible point with respect to reliability. Our main design philosophy is that the switch should be able to continue operating, as much as possible, even with the failed controller and providing a mechanism with which a standby controller can gain the control of the switch. When a standby controller takes over the control of switch, it needs to recover the state of primary controller just before its crash. Primitives of these operations are included in ONSCI. Our design also allows to take the controller down in a graceful manner so as to allow upgrading or fixing the control software.

\section{MODELS AND STRUCTURE}

The switch interface design is based on conceptual modeling of the switch behavior as well as the way it supports different QoS requirements. These models cover most of the switch implementations regardless of the way the basic switching function is implemented, as well as the ways buffers are managed across different connections, and the way QoS is provided. It also captures a broad range of the switch core control architecture.

\subsection{THE SETTING}

ONSCI is defined in terms of AAL5 message exchanges between the switch and a controller. This interconnection may use pre-configured ATM VCs on a regular ATM port, or a virtual circuit between the switch and the controller as shown in Figure 3. This allows a single controller to control multiple switches as depicted in Figure 3. The switch controller controls switch-1 directly (i.e., the control VC indicated by the dot-dashed line) and controls switch-2 indirectly (i.e., the virtual circuit indicated by the dashed line).

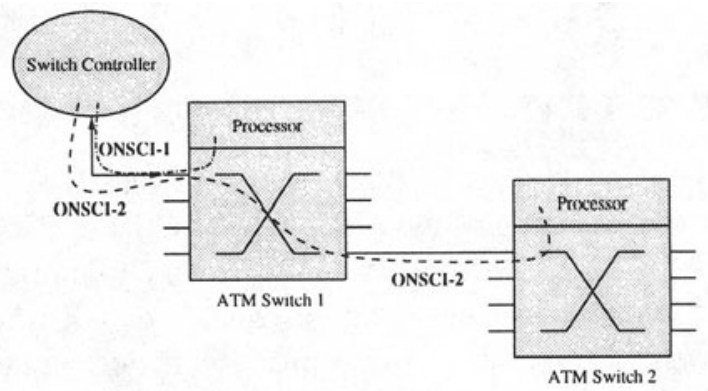

Figure 3 Control of multiple switches 


\subsection{PROTOCOL NATURE}

The interaction between the switch controller and the switch is master-slave. The controller issues a command to the switch by sending a request packet which is encapsulated in an AAL5 frame. The switch performs the request and send a response message with a similar structure. Every packet contains a 32-bit transaction identifier which is used by the controller to match a response to a request. Most of the request messages are idempotent, and it is the responsibility of the controller to re-issue requests in case of packet losses. The resource management messages are not idempotent and carry another identifier. The state of one resource management packet is remembered by the switch to detect retransmissions.

Other than these messages, the switch asynchronously sends event messages to the controller to report certain conditions like a link going down, in a manner similar to that described in [14].

\subsection{CONNECTIONS}

The switch and its controller refer to individual connections by means of an abstract data structure which is referred to as generalized forwarding table. An entry in the generalized forwarding table consists of a set of input designators, a set of output designators, a traffic descriptor and some additional parameters. An I/O designator is a triplet of port number, VP and VC identifiers and is the means by which the switch identifies individual data flows.

Every cell arriving at any input designator are switched to all the corresponding output designators. The traffic descriptor follows the ATM Forum's specification and includes traffic type and other parameters (see [7]). An entry in the generalized forwarding table defines a flow in one direction only. A bidirectional connection includes two entries, one in each direction. The port to which the controller is attached treated as other ports and cells can be forwarded to and from it just like other ports.

\subsection{SWITCH PARAMETERS AND QOS MODEL}

The switch can support a set of predefined QoS classes. The set can vary from the minimum set defined by the ATM Forum (CBR, VBR-RT, VBR-NRT, ABR, UBR) [18] to a larger set which is proprietary to an individual switch. The switch has its own proprietary way to translate the class identity and call rate into its internal switch tables. This translation is only known to the switch and may be hidden from the controller. Sometimes this translation is easy as mapping the given QoS class into a internal priority class and including rate parameters for switch policing functions. Other more sophisticated switches need to translate the rate parameters and insert these translation into switch 
internal buffer management table. The translation to the internal format is done by the embedded processor on the switch.

\subsection{SWITCH ACCOMMODATION CONTROL MODEL}

In addition to the ability of the switch to carry calls of certain QoS and rates, we assume that an accommodation test mechanism also exists by which the ability of the switch to accommodate an additional connection can be checked.

At the source of a connection a routing decision must be made, and the source node must be able to estimate the ability of every switch along the computed route to accommodate the requested connection (we refer to this as the remote CAC test). During connection set-up, every node along the path must verify that the resources required by the connection are available (we refer to this as the local CAC test). Furthermore, there are two phases during the set-up time: first the node checks for resource availability and sets the resource aside tentatively, and then, when all nodes along the path have confirmed the availability of the resources a commitment is made.

The local CAC test described above, is done by the local controller in cooperation with the local switch. The accommodation test must be conservative in the sense that whenever it indicates that a call can be accommodated, it should be able to obey the QoS contract in the physical switch. On the other hand, the accommodation control test should be fairly accurate and not overly conservative to allow for efficient utilization of all the physical resources available.

In order to approximate remote CAC, a generic call admission control (GCAC) procedure has been defined which takes the new call rate parameters (SCR, PCR, MBS) along with the three parameters (ACR, CRM, VF) which are advertised by remote node for each of its link. The advertised GCAC parameters are switch dependent (VF parameter in PNNI GCAC), and the controller should be able to interrogate the local switch regarding the availability of resources (VF parameter).

The above observations have led to a CAC model whose major components are shown in Figure 4. The translator block converts commands which are expressed in terms of a generalized VC table entry to the switch internal formats and parameters. In the reverse direction, the translator will make some of its internal settings available to the controller. The accommodation test and availability checks are performed with the accommodation oracle whose components are depicted in Figure 5.

At the core of the accommodation oracle is a function or a performance model which can estimate quite accurately whether a new call of given QoS and rate parameters can be accommodated. While such a procedure is an essential block in any ATM system, it is the most switch dependent one. Even if we use known 


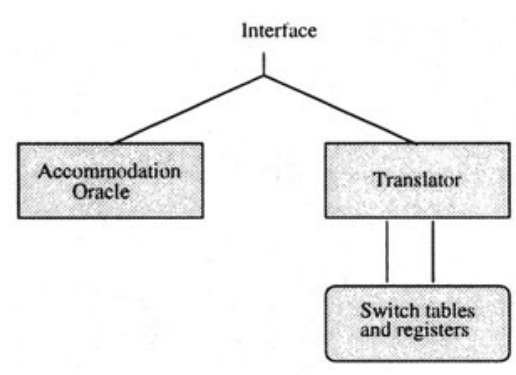

Figure 4 Components of accommodation mechanism

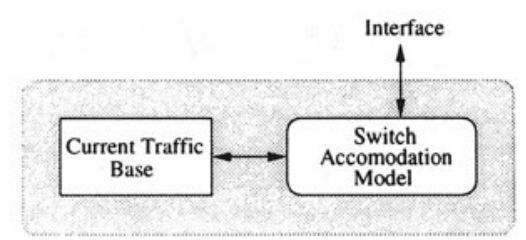

Figure 5 Structure of accommodation oracle

models (such as one of the equivalent capacity models of [18], [8] or [4]) there are necessarily some parameters in these models which are switch specific and capture, for example, the amount and structure of buffering in the switch. It is quite possible that the implementation of the oracle might contain a proprietary switch model. In addition the oracle maintains a current traffic base, whose structure is undefined but which embodies the oracle's view of existing traffic base. To make an accommodation decision the oracle combines its notion of the current traffic with the requested new connection.

With no further assumptions, the traffic base will consist of the list of connections with their traffic descriptors and QoS parameters. This will render the test and translate operations very complex and time consuming, adversely impacting the connection set-up procedure as we described earlier. To facilitate these operations we further assume that the accommodation control function is additive (or cumulative). This means that if $A$ is the set of existing calls over a link of the switch and $c_{1}$ and $c_{2}$ are two given calls, and if $c_{1}$ is feasible under link state $A \cup c_{2}$, then $c_{2}$ is feasible under the link state $A \cup c_{1}$. This implies that the list of individual connections need not be maintained but that some form of aggregation would suffice and that allocating and de-allocating resources can be easily incorporated into the base. We also assume that the accommodation control function is monotonic which means that if $c_{1}$ is feasible under state $A$ and $c_{2}$ has a rate description (and QoS) that is smaller than that of $c_{1}$, then $c_{2}$ is also feasible under state $A$. With this assumption one availability check would suffice to quickly determine the availability of resources for several (small) connections.

If implemented in the switch, the accommodation oracle and the translator have direct access to all the switch registers and can make the most accurate calculations. If implemented in the controller, the accommodation test function can be more efficient as the oracle can make full use of the computational resources of the controller and does not need to communicate with the switch for each decision. In this case the oracle may have to retrieve switch-specific 
information from the switch, for which the private primitive is provided in the interface.

\section{SWITCH RELIABILITY SUPPORT}

The switch controller which has a large software component is more prone to failure than the ATM switch. ONSCI primitives allow a secondary controller located anywhere in the retwork, to replace the failed controller of a switch, enhancing the controller's availability. The controller is not involved in normal data transfer operation, and therefore the connections successfully established before its failure are not affected by its failure.

As soon as the failure is detected, a secondary controller sets-up VCs to the switch and modifies its forwarding table (using a pre-established virtual circuit) to divert the special VCs at each of its ports. It then queries the ATM switch to get a list of connections in progress and gathers information from its neighbours to reconstruct the state of primary controller and starts working as the primary controller of the switch. The following sections describe these operations in more detail.

\subsection{MULTIPLE CONTROLLERS OF A SWITCH}

A control point is a special I/O designator on which incoming messages are treated as ONSCI requests by the switch and the outgoing messages contain the corresponding switch response. Every switch has at least one default control point, which is used by the switch to locate its primary controller when the switch is powered up.

Secondary controllers may be attached to the switch by dynamically creating new control points (using ONSCI primitives) and creating a VC from the new control point to the secondary controller. Thus, a controller can, in principle control, more than one ATM switch at a time as indicated earlier in Figure 3. In a realistic setting, a primary controller of a switch may also act as a secondary controller for another switch.

\subsection{REBUILDING STATE INFORMATION}

When a secondary controller takes over the control of an ATM switch, it first rebuilds the state of the primary controller, then it creates an additional secondary controller (if possible) and finally advertises its presence in the network and starts working as the primary controller of the switch. Of these, the first one is the hardest. The relevant state of the primary controller includes list of connections (in the generalized VC table), traffic descriptor and QoS of individual connections, and other information like link status of individual link, the load on ATM switches etc. The secondary controller gathers the 
relevant information by three methods: from periodic broadcasts, by querying neighbors, and by querying switch.

The list of active connections on a port along with the VCI, traffic descriptor and QoS of the connections can be obtained from neighbors. This along with the forwarding information from the switch's cross-connect is sufficient for building the generalized forwarding table. State of the traffic base of accommodation control oracle is constructed by accumulating the traffic descriptor and QoS of each connection to the traffic base. Recall that in Section 3.5 we assumed that the accommodation control procedure is cumulative. Therefore the connections can be re-accumulated in any order to reconstruct the traffic base.

The state information thus constructed reflects the state of the controller at the time it crashed. Some events (like connection tear-down) may have occurred between the crash and subsequent recovery. Thus, the recovering controller needs to carry out corresponding update in its state and possibly some additional operations, described next.

\subsection{PARTIAL AND ZOMBIE CONNECTIONS}

A partial connection is a connection such that there is one neighboring controller which believes that the connection exists and there is another which believes it has been closed. A zombie connection is one where none of the adjacent controllers believes that the connection exists. A connection may become partial or zombie if its tear-down is initiated after crash of a controller in its path.

The recovering controller identifies the deleted input and output branches, traffic descriptor and QoS of partial connections, using the neighbour and switch information. It then removes these branches from the switch forwarding table, updates the current traffic base and initiates a connection tear-down if needed.

Zombie connections are more difficult to deal with. Their existence can be identified but their traffic descriptors and QoS parameters may not be recoverable. In order to reclaim the resources allocated to zombie connections, the entire traffic base may be rebuilt from scratch.

The overall availability of the network to incoming calls mainly depends upon the number of secondary controllers, the mean time taken by a secondary controller in rebuilding its state and the mean time taken to repair the faulty controller. From these parameters, using the standard reliability theory techniques [2], [17] it should be possible to figure out the (approximate) number of secondary controllers needed to ensure a given availability.

The primitives in ONSCI protocol provide support needed by the controller from the switch in order to make the network tolerant to faults in the controller. For the details of the protocol, the reader is referred to [7]. With proper design 
of protocols at higher layer, and support from ONSCI it is possible to build a fault tolerant network.

\section{DISCUSSION AND CONCLUSION}

We have proposed the OpeNet Switch Control Interface (ONSCI), a new open ATM switch control interface based upon principles of distributed systems. ONSCI is switch independent and supports a variety of primitives to allow many network control platforms to be implemented on top of it. We chose the ATM Forum's traffic management model and the OpeNet signalling as an example.

Two innovative contributions are included in ONSCI: (1) support for a resource management scheme for the provision of general QoS that is both switch and signaling platform independent (2) support for a fault tolerant operation in the vein of increasing availability. Our design assumes limited switch capability in terms of the amount of memory and particularly CPU power. The idea behind this design is to allow simple (read: stable and reliable) processor as part of the switch.

One of the major problem in designing this interface was how to identify resources of the switch allocated to a particular connection. Imagine a situation when a branch is to be added to an existing multicast connection. In order to compute the amount of additional resources needed for this purpose, the resources already allocated (buffers, bandwidth, etc.) must be known. The CPU of the switch performs only very simple operations and is incapable of storing this resource mapping. The controller cannot store this information either as the amount and structure of switch resources is switch specific which cannot be generalized.

Resource identification has other benefits also. If the switch resources allocated to a particular connection were identifiable, all the commands in the resource management group could have been made idempotent, simplifying the protocol. The problem of reclaiming resources from zombie connections would have become much easier.

Explicit resource identification requires enormous book-keeping in the switch (and a more complicated switch model). It also needs significant software on the switch. This conflicts with our original goal of decoupling software and hardware components of a traditional ATM switch, distributed implementation of these two components, and open switch control interface. Despite the advantages of explicit resource identification, we decided not to opt for it. The tradeoff in favor of simple switch software is desirable.

We solved the resource identification issues in a variety of ways. In terms of the resource management commands, we chose to implement those in a more reliable manner which includes specific ACKs for each request. In terms of 
resource searching, we require that all messages that refer to resources should carry enough information to allow the switch to infer, rather than compute or search, the resources involved (e.g., including all I/O designators in identifying resources of a multicast connection). This is, in addition to the assumption that resource accommodation is monotonic and cumulative.

The inability to identify resources impacts the recovery process after failures. To that end we provided primitives that will allow to reconstruct the state of a failed controller, considering the fact that a large amount of information can be acquired from neighboring controllers.

ONSCI was implemented and integrated with the OpeNet control platform. Pure ONSCI implementation on an ATM switch was not available, but we had access to GSMP-capable ATM switch. We wrote a thin layer of software which converts ONSCI message to GSMP messages and GSMP response to ONSCI response. Using this basic functionality of ONSCI, like connection management, statistics etc., were successfully tested.

\section{Acknowledgments}

The authors thank Israel Cidon and Moshe Sidi for their valuable comments and suggestions while designing the protocol. Thanks to Amit Gupta who provided much needed help in design as well as implementation phase.

\section{References}

[1] C. M. Adam, A. A. Lazar, and M. Nandikesan. QOS extensions to GSMP. Technical Report 471-97-05, Center for Telecommunications Research, Columbia University, New York, April 1997.

[2] E. Balaguruswamy. Reliability Engineering. Tata McGraw Hill, New Delhi, India, 1984.

[3] I. Cidon, T. Hsiao, P. Jujjavarapu, A. Khamisy, A. Parekh, R. Rom, and M Sidi. The OpeNet architecture. Technical Report SMLI-TR-95-37, Sun Microsystems Laboratories, Mountain View, CA, USA, December 1995. http://www.sunlabs.com/technical-reports/1995/smli_tr-95-37.ps.

[4] A. Elwalid and D. Mitra. Effective bandwidth of general markovian traffic and admission control of high speed networks. IEEE/ACM Transactions on Networking, 1:329-343, 1993.

[5] Joe Evans. ATMF extensions to GSMP. In Proceedings of OPENSIG Fall '97 Workshop: Open Signalling for ATM, Internet and Mobile Networks, Columbia University, New York, NY, October 1997.

[6] S. Floyd and V. Jacobson. Link-sharing and resource management models for packet networks. IEEE/ACM Transactions on Networking, 3(4):365386, August 1995. 
[7] R. Garg and R. Rom. The OpeNet switch control interface specification. Technical Report SMLI TR-97-0543, Sun Microsystems Laboratories, Mountain View, CA, USA, November 1997.

[8] L. Georgiadis, R. Guerin, V. Peris, and K. Sivarajan. Efficient QoS provisioning based on per node traffic saping. IEEE/ACM Transactions on Networking, pages 482-501, 1996.

[9] J. Hyman, A. Lazar, and C. Pacifi. Real-time scheduling with quality of service constraints. IEEE Journal on Selected Areas of Communications, pages 1052-1063, September 1991.

[10] IEEE. Proposed IEEE standard for application programming interfaces for networks. http://www.iss.nus.sg/IEEEPIN/.

[11] A. A. Lazar, S. Bhonsle, and K.S. Lim. A binding architecture for multimedia networks. Journal of Parallel and Distributed Systems, 30(2):204 216, November 1995.

[12] A. A. Lazar and Franco Marconici. Towards an API for ATM switch control. Technical Report CU/CTR/TR 441-96-07, Center for Telecommunications Research, Columbia University, April 1996. http://www.ctr.columbia.edu/comet/xbind/xbind.html.

[13] K. Van Der Merwe and Ian Leslie. Switches and dynamic virtual ATM networks, July 1996. Computer Laboratory, University of Cambridge.

[14] P. Newman, W. Edwards, R. Hinden, E. Hoffman, F. Ching Liaw, T. Lyon, and G. Minshall. Ipsilon's genaral switch management protocol, August 1997. RFC 1987.

[15] P. Newman, W. Edwards, R. Hinden, E. Hoffman, F. Ching Liaw, T. Lyon, and G. Minshall. Ipsilon's general switch management protocol specification version 2.0, March 1998. RFC 2297.

[16] P. Newman, G. Minshall, and T.L. Lyon. IP switching-ATM under IP. IEEE/ACM Transactions on Networking, 6(2):117-29, April 1998.

[17] Charles O Smith. Introduction to Reliability in Design. McGraw Hill, NY, 1976.

[18] The ATM Forum. Private network-network specification interface v1.0 (PNNI 1.0). Technical Report af-pnni-0055.000, The ATM Forum, March 1996.

[19] L. Zhang, S. Deering, D. Estrin, S. Shenker, and et. al. RSVP: a new resource reservation protocol. IEEE Network, 7(5):8-18, September 1993. 